\title{
Detection of the basement membrane-degrading proteolytic activity of Paracoccidioides brasiliensis after SDS-PAGE using agarose overlays containing Abz-MKALTLQ -ED Dnp
}

\section{R. Puccia ${ }^{1}$, M.A. Juliano${ }^{2}$, L. Juliano ${ }^{2}$, L.R. Travassos ${ }^{1}$ and A.K. Carmona ${ }^{2}$}

Departamentos de ${ }^{1} \mathrm{M}$ icrobiologia, Imunologia e Parasitologia and

${ }^{2}$ Biofísica, Universidade Federal de São Paulo, São Paulo, SP, Brasil

\section{Correspondence \\ R. Puccia \\ Departamento de Microbiologia, Imunologia e Parasitologia, UNIFESP Rua Botucatu 862,80 andar 04023-062 São Paulo, SP Brasil \\ Fax: +55-11-571-5877 \\ E-mail: rosana.dmip@epm.br}

Presented at the 5th Brazilian Symposium on Extracellular Matrix - SIMEC, Angra dos Reis, RJ, Brasil, September 7-10, 1998.

Research supported by FAPESP (No. 1995/0559-8), BID-FINEP, Pronex and $\mathrm{CNPq}$

\section{Abstract}

We have characterized, in the Paracoccidioides brasiliensis yeast phase, an exocellular SH-dependent serine proteinase activity against Abz-MKRLTL-EDDnp and analogous fluorescent-quenched peptides, and showed that it is also active against constituents of the basement membrane in vitro. In the present study, we separated the components of $P$. brasiliensis culture filtrates by electrophoresis and demonstrated that the serine-thiol exocellular proteinase has a diffuse and heterogeneous migration by SDS-PAGE, localizing in a region between 69 and $43 \mathrm{kDa}$. The hydrolytic activity was demonstrable after SDS-PAGE using buffered agarose overlays of Abz-MKALTLQ-EDDnp, following incubation at $37^{\circ} \mathrm{C}$, and detection of fluorescent bands with a UV transilluminator. Hydrolysis was more intense when incubation was carried out at basic $\mathrm{pH}$, and was completely inhibited with $2.5 \mathrm{mM}$ PMSF and partially with sodium 7-hydroxymercuribenzoate $(2.5 \mathrm{mM}$ $p$-HMB), suggesting its serine-thiol nature. A proteolytic band with similar characteristics was observed in conventional gelatin zymograms, but could not be correlated with a silver-stained component. Detection of the serine-thiol proteinase in substrate gels after SDSPAGE provides a useful way of monitoring purification of the basement membrane degrading enzyme.

Paracoccidioides brasiliensis is a dimorphic fungus that causes human paracoccidioidomycosis (PCM), a systemic mycosis endemic in Latin America, which can be fatal in immunocompromised individuals if not successfully treated. Patients with PCM can have multiple clinical forms which are dependent on the relationship between the host's

\section{Key words}

- P. brasiliensis

- Serine-thiol proteinase

- SD S-PAGE

- Fluorescent-quenched peptides resistance mechanisms and fungal pathogenicity (1). The yeast cell wall $\alpha$-glucan (2) and the gp43 antigen $(3,4)$, which is protective in experimental murine PCM (5), have been implicated in fungal virulence. Other virulence factors in $P$. brasiliensis probably exist.

We have characterized a subtilisin-like, 
SH-dependent serine proteinase activity in $P$. brasiliensis grown in the yeast phase (6). Internally quenched fluorescent peptides derived from MKRLTL and flanked by Abz (ortho-aminobenzoyl) and EDDnp (ethylenediaminedinitrophenyl) were cleaved at the L/T bond when incubated with a $P$. brasiliensis culture filtrate. The exocellular proteinase activity had an optimum $\mathrm{pH}$ of $>9.0$ and was irreversibly inhibited by PMSF, mercuric acetate and sodium 7-hydroxymercuribenzoate ( $p$-HMB). Moreover, the enzyme was able to selectively cleave, in vitro, components of the basal membrane or associated with it, including laminin, fibronectin, type IV collagen and proteoglycans. Such activity could be of biological significance in tissue invasion by the fungus (7).

The serine-thiol proteolytic activity involves an enzyme of very high specific activity, which is rather unstable and is lost after several chromatographic steps. So far, a single silver-stainable band in SDS-PAGE has not been observed in fractionated preparations, which has hampered the purification and characterization of the enzyme. The objective of the present study was to detect the exocellular serine-thiol proteinase activity in SDS-PAGE gels using overlays of AbzMKALTLQ-EDDnp. This is a substrate analogous to Abz-MKRLTL-EDDnp but with a better $V_{\max } / K_{\mathrm{m}}$ value (6), and was synthesized as described previously $(8,9)$. A conventional gelatin zymogram was used in parallel.

P. brasiliensis strain 339 (originally provided by Dr. Angela Restrepo-Moreno, Medellin, Colombia) was cultivated at $35^{\circ} \mathrm{C}$, with shaking, in modified YPD $(0.5 \%$ Bactoyeast extract, $0.5 \%$ casein peptone and $1.5 \%$ glucose), and the culture supernatant was collected by paper filtration at the peak of proteinase activity against Abz-MKALTLQEDDnp (7). Ammonium sulfate was added to the culture filtrate at $40 \%$ saturation and the precipitate separated by centrifugation (16300 $\mathrm{g}, 30 \mathrm{~min})$. The supernatant $(50 \mathrm{ml})$ was concentrated by FPLC in a Phenyl Superose HR 5/5 (Pharmacia/LKB, Uppsala, Sweden) column, according to standard procedures. The proteolytic activity against AbzMKALTLQ-EDDnp was concentrated in two fractions, denoted F3 and F4, which were analyzed on substrate gels. This concentration procedure using Phenyl Superose, although more laborious, resulted in a better yield of the active enzyme than precipitation of culture filtrates with $50 \%$ ammonium sulfate (6), since very little of the hydrolytic activity is precipitated with salt.

For SDS-PAGE analysis, F3 and F4 were electrophoresed $\left(100 \mathrm{~V}\right.$, at $\left.4^{\circ} \mathrm{C}\right)$ in conventional linear $10 \%$ gels (10), using sample buffer containing SDS at $0.5 \%$ final concentration without boiling. Pre-stained low molecular weight protein markers (Gibco BRL, Gaithersburg, MD, USA) were applied to the gels, and their migration position was marked with a needle after running as a reference for localization of the proteolytic bands. The gels were then washed for one hour in $0.05 \mathrm{M}$ Tris- $\mathrm{HCl}, \mathrm{pH} 8.5,10 \% \mathrm{MeOH}$ (11), with three changes, and finally in buffer without MeOH. Abz-MKALTLQ-EDDnp $(100 \mu \mathrm{g} / \mathrm{ml})$ overlays were prepared in $1 \%$ buffered agarose $(0.75 \mathrm{~mm}$ thick), placed on the washed SDS-PAGE gels, and incubated at $37^{\circ} \mathrm{C}$ in a moist chamber. Fluorescent green bands were visualized with a UV transilluminator $(302 \mathrm{~nm})$ and the results were recorded on Polaroid films using a yellow filter.

Figure 1A shows the fluorescence obtained after increasing incubation times with Abz-MKALTLQ-EDDnp in agarose overlays. The proteolytic activity appeared as a diffuse zone in the gel between bovine serum albumin (BSA) and egg ovalbumin (OVA), or between 69 and $43 \mathrm{kDa}$ apparent molecular masses. The fluorescent band at $43 \mathrm{kDa}$ (gp43) seen in F4 was not due to proteolysis, but to natural fluorescence emitted by the molecule, and could still be seen without the peptide overlay, or when the 
sample was previously boiled in SDS for protease inactivation (data not shown). The protein profiles of F3 and F4 (Figure 2A) indicated that $\mathrm{F} 4$ was rich in the gp 43 component, which could barely be seen in F3 by silver staining. This fractionation after Phenyl Superose chromatography has been advantageous in that only F3 fractions are now being processed for proteinase purification. This procedure avoids the gp43 depletion step by affinity chromatography which was used before (6). On the other hand, F3 is usually twice more active than F4 against Abz-MKALTLQ-EDDnp in solution.

In the experiment shown in Figure 1A, maximum fluorescence arising from the hydrolyzed substrate was achieved after $1 \mathrm{~h}$ and $45 \mathrm{~min}$ of incubation at $37^{\circ} \mathrm{C}$. After $3 \mathrm{~h}$, it diffused and was not as intense. The fluorescence intensity and the incubation time necessary to reach a maximum of fluorescence varied with the activity of the sample in a dose-dependent fashion (data not shown). The result shown in Figure 1A was similar when the samples were incubated with reduced or nonreduced SDS-PAGE sample buffer.

The specificity of the activity against AbzMKALTLQ-EDDnp in agarose overlays was assessed by pre-incubation (30 $\mathrm{min}$, room temperature) of $\mathrm{F} 3$ with known protease inhibitors before addition of the sample buffer and electrophoresis. The result is illustrated in Figure 1B, where proteolysis was completely inactivated by $2.5 \mathrm{mM}$ PMSF (lane 4 ), and by boiling in SDS buffer (lane 2); $p$ $\mathrm{HMB}(0.15$ and $2.5 \mathrm{mM})$ was partially inhibitory, especially at higher concentrations (lane $\left.7^{*}\right)$. Since this is not an active site inhibitor, it could have been partly dissociated during electrophoresis. In addition, the activity was more intense upon incubation at $\mathrm{pH} 8.5$ than at $\mathrm{pH} 6.5$ (data not shown). These results strongly suggest that the exocellular serinethiol activity previously characterized in $P$. brasiliensis (6) is due to a proteinase which migrates diffusely between 69 and $43 \mathrm{kDa}$ in

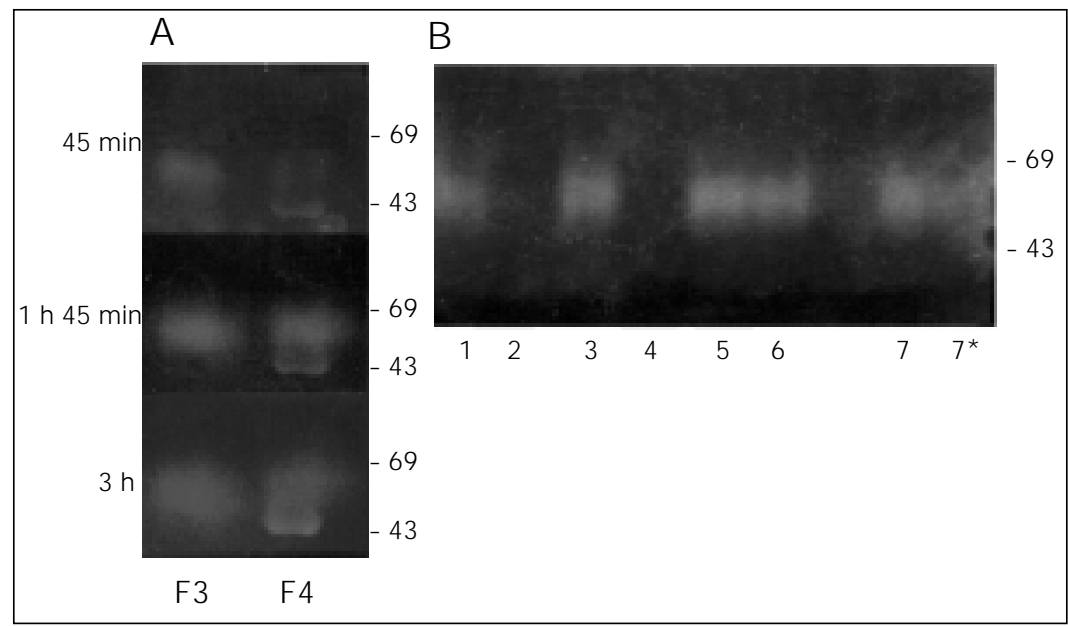

Figure 1 - Demonstration of P. brasiliensis exocellular proteolytic activity in SDS-PAGE gels using Abz-MKALTLQ-EDDnp in agarose overlays, and enzyme characterization with protease inhibitors. A, The hydrolytic activity in $\mathrm{F} 3(1.25 \mu \mathrm{g})$ and $\mathrm{F} 4(5.0 \mu \mathrm{g})$ was recorded after $45 \mathrm{~min}, 1 \mathrm{~h}$ and $45 \mathrm{~min}$, and $3 \mathrm{~h}$ of incubation at $37^{\circ} \mathrm{C}$. Fluorescence of the $43-\mathrm{kDa}$ component was intrinsic, and was observed without the peptide overlay. B, F3 (1.25 $\mu \mathrm{g})$ alone (lane 1), or inactivated by boiling (lane 2), pre-incubated with $2.5 \mathrm{mM}$ ortho-phenanthroline and $5 \mathrm{mM}$ EDTA (lane 3), $2.5 \mathrm{mM}$ PMSF (lane 4), $0.13 \mathrm{mM}$ trans-epoxy-succinyl-Lleucylamido-(4-guanido)-butene, E-64 (lane 5), 2.5\% DMSO (lane 6), 0.15 mM (lane 7) and $2.5 \mathrm{mM}$ (lane $7^{*}$ ) p-HMB. Molecular mass markers $(\mathrm{kDa})$ are indicated on the right side.

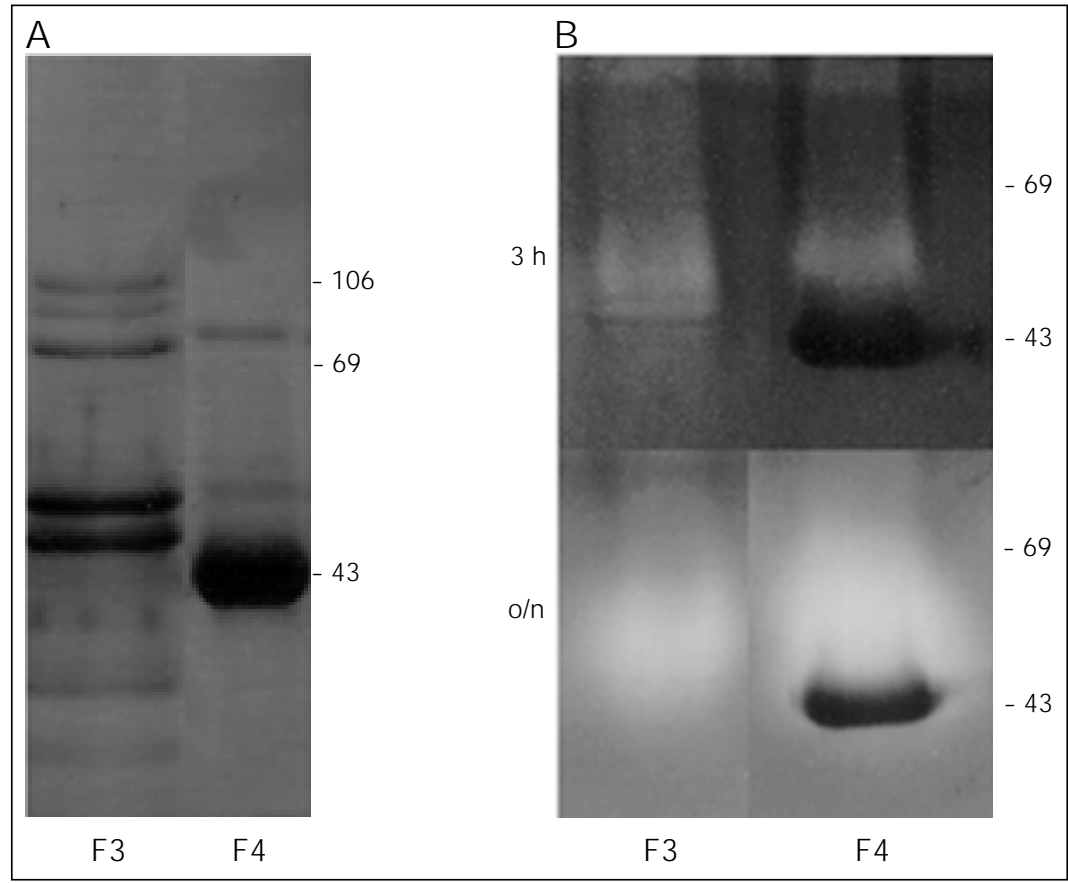

Figure 2 - Silver-stained SDS-PAGE (A) and zymogram (B) profiles of $F 3$ and $F 4$ fractions of $P$. brasiliensis exocellular components. For the zymograms, the polyacrylamide gels were impregnated with $0.1 \%$ gelatin. After the electrophoretic run, the gels were washed with buffered $\mathrm{MeOH}$, as indicated in the text, and incubated at $37^{\circ} \mathrm{C}$ in $50 \mathrm{mM}$ Tris- $\mathrm{HCl}, \mathrm{pH} 8.5$, for $3 \mathrm{~h}$ or overnight $(\mathrm{o} / \mathrm{n})$, as indicated. Proteolytic bands were visualized by staining with Coomassie brilliant blue. The amounts loaded onto the gels were: $0.5 \mu \mathrm{g}$ of F3 and $1 \mu \mathrm{g}$ of F4 for silver staining and $5 \mu \mathrm{g}$ of F4, $1.25 \mu \mathrm{g}(3 \mathrm{~h})$ or $0.25 \mu \mathrm{g}(\mathrm{o} / \mathrm{n})$ of F3 for zymograms. Molecular weight markers $(\mathrm{kDa})$ are indicated on the right side. 
SDS-PAGE gels. This is also in accordance with our previous data (6) showing that the exocellular serine-thiol activity against AbzMKRLTL-EDDnp was eluted in a single peak near ovalbumin. Furthermore, the diffuse SDS-PAGE migration of the proteinase is probably due to heterogeneous and/or abundant glycosylation, as inferred from binding of the enzymatic activity to concanavalin A (Puccia R and Carmona AK, unpublished results).

The same results were obtained when F3 and F4 were analyzed for gelatinase activity in a zymogram (Figure 2B), where a fuzzy proteolytic band between 69 and $43 \mathrm{kDa}$ could be seen even after $3 \mathrm{~h}$ of incubation at $37^{\circ} \mathrm{C}$. After overnight incubation, the proteolytic zone was very clear and broad. The proteolysis inhibition pattern and the $\mathrm{pH}$ dependence in zymograms were similar to those with Abz-MKALTLQ-EDDnp in agarose overlays. Additional results obtained with gelatin zymograms showed that the serine-thiol proteinase activity was not altered by heating at $45^{\circ} \mathrm{C}$ or by lyophilization, and could not be detected after incubation at room temperature (data not shown). Thermostability was checked in protein-rich preparations due to the unstable nature of the proteinase in more purified fractions.

Gelatinase activity in culture filtrates of $P$. brasiliensis concentrated by heat evapo- ration at $45^{\circ} \mathrm{C}$ and lyophilization has been reported (12). For strain 339, the authors showed what seems to be two overlapping broad bands between 43 and $78 \mathrm{kDa}$. This gelatinase activity may have been due to the serine-thiol proteinase, on the basis of similarity in molecular weight and heat-stability. However, this is probably not the case, since that activity was very strong upon incubation at room temperature. However, it is unlikely that the gelatinase activity mentioned above (12) was due to gp43, as attributed by the authors without any evidence, since there were a number of components migrating in the same broad region of the proteolytic zone. Moreover, protease motifs have not been reported in the gp43 gene sequence (13) and the proteolytic properties associated with gp43 in the past (14) were later shown to be due to aggregation (6).

In the present work, we show for the first time the usefulness of internally quenched fluorescent peptides flanked by Abz (orthoaminobenzoyl) and EDDnp (ethylenediaminedinitrophenyl) in agarose overlays for the detection of proteolytic activities after SDS-PAGE. For $P$. brasiliensis serine-thiol proteinase, visualization of the activity zone in substrate gels is essential for further characterization and purification of the basement membrane degrading enzyme.

\section{References}

1. Franco M (1987). Host-parasite relationships in paracoccidioidomycosis. J ournal of Medical and Veterinary Mycology, 25: 5-18.

2. San-Blas G \& San-Blas F (1994). Biochemistry of Paracoccidioides brasiliensis dimorphism. In: Franco M, Lacaz CS, Restrepo-Moreno A \& Del Negro C (Editors), Paracoccidioidomycosis. CRC Press, Boca Raton.

3. Vicentini AP, Gesztesi J L, Franco MF, Souza W, Moraes JZ, Travassos LR \& Lopes J D (1994). Binding of Paracoccidioides brasiliensis to laminin through surface glycoprotein gp43 leads to enhancement of fungal pathogenesis. Infection and Immunity, 62: 1465-1469.

4. Gesztesi J L, Puccia R, Travassos LR, Vicentini AP, Franco MF \& Lopes JD (1996). Monoclonal antibodies against the 43,000 Da glycoprotein from Paracoccidioides brasiliensis modulate laminin-mediated fungal adhesion to epithelial cells and pathogenesis. Hybridoma, 15: 415422.

5. Taborda CP, J uliano MA, Puccia R, Franco $M \&$ Travassos LR (1998). Mapping of the T-cell epitope in the major 43-kilodalton glycoprotein of Paracoccidioides brasiliensis which induces a Th-1 response protective against fungal infection in BALB/C mice. Infection and Immunity, 66: 786-793.
6. Carmona AK, Puccia R, Oliveira MCF, Rodrigues $\mathrm{E}$, J uliano $\mathrm{L} \&$ Travassos LR (1995). Characterization of an exocellular serine-thiol proteinase activity in Paracoccidioides brasiliensis. Biochemical J ournal, 30: 209-214.

7. Puccia R, Carmona AK, Gesztesi J L, J uliano L \& Travassos LR (1998). Exocellular proteolytic activity of Paracoccidioides brasiliensis: cleavage of components associated with the basement membrane. Medical Mycology, 36: 345348.

8. Chagas J R, J uliano L \& Prado ES (1991). Intramolecularly quenched fluorogenic tetrapeptide substrates for tissue and 
plasma kallikreins. Analytical Biochemistry, 192: 419-425.

9. Oliveira MCF, Hirata IY, Chagas JR, Boschcov P, Gomes RAS, Figueiredo AFS \& J uliano L (1992). Intramolecularly quenched fluorogenic peptide substrates for human renin. Analytical Biochemistry, 203: 39-46.

10. Laemmli UK (1970). Cleavage of structural proteins during the assembly of the head of bacteriophage T4. Nature, 227: 680-685.

11. Lundy FT, Magee AC, Blair IS \& MCDwell DA (1995). A new method for detection of proteolytic activity in Pseudomonas lundensis after sodium dodecyl sulfatepolyacrylamide gel electrophoresis. Electrophoresis, 16: 43-45.

12. Vaz CAC, Mackenzie DWR, Hearn V, Camargo ZP, Singer-Vermes LM, Burger E \& Calich VLG (1994). Gelatinase activity of exoantigens from virulent and non-virulent isolates of Paracoccidioides brasiliensis. J ournal of Medical and Veterinary Mycology, 32: 65-69.

13. Cisalpino PS, Puccia R, Yamauchi LM, Cano MIN, Silveira JF \& Travassos LR (1996). Cloning and characterization of the gene encoding the major diagnostic antigen of Paracoccidioides brasiliensis: expression of epitopes of the $43,000 \mathrm{Da}$ glycoprotein recognized by antibodies from human patients. J ournal of Biological Chemistry, 271: 4553-4560.

14. Puccia R \& Travassos LR (1991). The 43$\mathrm{kDa}$ glycoprotein from Paracoccidioides brasiliensis and its deglycosylated form: excretion and susceptibility to proteolysis. Archives of Biochemistry and Biophysics, 289: 298-302. 